\title{
Demethylation of the MafB promoter in a compromised $\beta$-cell model
}

\author{
Wataru Nishimura, ${ }^{1,2}$, Naoko Ishibashi', Koki Eto', Nobuaki Funahashi', \\ Haruhide Udagawa', Harukata Miki ${ }^{2}$, Souichi $\mathrm{Oe}^{2}$, Yasuko Noda ${ }^{2}$ and Kazuki Yasuda' \\ ${ }^{1}$ Department of Metabolic Disorders, Diabetes Research Center, Research Institute, National Center for Global \\ Health and Medicine, Tokyo 162-8655, Japan \\ ${ }^{2}$ Division of Anatomy, Bio-imaging and Neuro-cell Science, Jichi Medical University, Shimotsuke, \\ Tochigi 329-0498, Japan
}

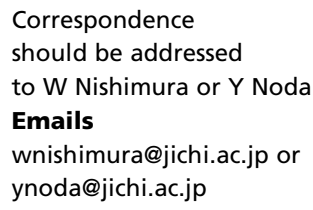

\begin{abstract}
Recent studies suggest that dedifferentiation of pancreatic $\beta$-cells is involved in compromised $\beta$-cell function in diabetes mellitus. We have previously shown that the promoter activity of $M a f B$, which is expressed in $\alpha$-cells of adult islets and immature $\beta$-cells in embryonic pancreas but not in mature $\beta$-cells in mice, is increased in compromised $\beta$-cells of diabetic model mice. Here, we investigated a rat $\beta$-cell line of INS1 cells with late-passage numbers, which showed extremely low expression of MafA and insulin, as an in vitro model of compromised $\beta$-cells. In these INS1 cells, the mRNA expression and the promoter activity of MafB were upregulated compared with the early-passage ('conventional') INS1 cells. Analysis of the MafB promoter in these late-passage INS1 cells revealed that specific CpG sites in the MafB promoter were partially demethylated. The reporter assay revealed that the unmethylated promoter activity of the 373 bp region containing these $\mathrm{CpG}$ sites was higher than the in vitro methylated promoter activity. These results suggest that the chronic culture of the rat $\beta$-cell line resulted in partial DNA demethylation of the MafB promoter, which may have a role in MafB promoter activation and possible dedifferentiation in our compromised $\beta$-cell model.
\end{abstract}

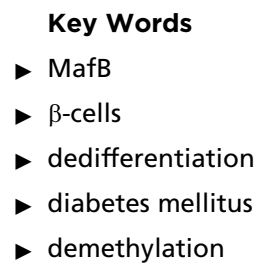

Journal of Molecular Endocrinology (2015) 55, 31-40

\section{Introduction}

Diabetes mellitus results from dysfunction of pancreatic $\beta$-cells, although the mechanism of impaired secretion of insulin from $\beta$-cells is not fully understood. Recent studies suggest that a subpopulation of $\beta$-cells in diabetic humans and mice undergo dedifferentiation rather than apoptosis. Upregulation of genes that are normally repressed in mature $\beta$-cells, including the transcription factors transiently expressed in endocrine progenitors of the embryonic pancreas, was identified in these compromised $\beta$-cells as a hallmark of dedifferentiation (Thorrez et al. 2011, Nishimura et al. 2015) and may result in the impaired insulin release in diabetes mellitus (Thorrez et al. 2011, Pullen \& Rutter 2013, Nishimura et al. 2015). However, the mechanism of the $\beta$-cell dedifferentiation remains unknown.

MafB, a b-Zip family transcription factor, is expressed in pancreatic $\alpha$-cells. MafB is also expressed in immature $\beta$-cells in embryonic pancreas, but not in mature $\beta$-cells in mice (Nishimura et al. 2006, Artner et al. 2010). Recent studies revealed that $M a f B$ is upregulated in the islets of MafA knockout (KO) mice, $\beta$-cell-specific FoxO1 KO mice with metabolic stress and conditional Pdx1 KO mice as a

Published by Bioscientifica Ltd 
hallmark of dedifferentiation (Talchai et al. 2012, Gao et al. 2014, Nishimura et al. 2015). We also reported that the promoter activity of MafB is increased in $\beta$-cells of diabetes model mice such as $d b / d b$ mice and mice that have received low-dose streptozotocin (Nishimura et al. 2015). Because MafB induces genome-wide changes in DNA methylation (Vicente-Dueñas et al. 2012), we speculated the MafB may be a key molecule for the upregulation of $\beta$-cell disallowed genes in dedifferentiated $\beta$-cells. Thus, we investigated the activity and methylation status of the $M a f B$ promoter using an in vitro model of compromised $\beta$-cells.

\section{Materials and methods}

\section{Cell culture}

The rat $\beta$-cell line of INS1 cells (Asfari et al. 1992) of the indicated passage (p) numbers were cultured in RPMI-1640 medium (Sigma) supplemented with 10\% (w/v) fetal bovine serum (FBS; Thermo Scientific, Rockford, IL, USA), $1 \mathrm{mM}$ sodium pyruvate, $10 \mathrm{mM}$ HEPES, $100 \mathrm{U} / \mathrm{ml}$ penicillin, $100 \mathrm{mg} / \mathrm{ml}$ streptomycin, and $55 \mu \mathrm{M}$ $\beta$-mercaptoethanol (Life Technologies) as described previously (Nishimura et al. 2013). HeLa cells (Scherer et al. 1953) were cultured in the DMEM (Sigma) supplemented with $10 \% \mathrm{FBS}, 100 \mathrm{U} / \mathrm{ml}$ penicillin, and $100 \mathrm{mg} / \mathrm{ml}$ streptomycin. All cell lines were cultured at $37^{\circ} \mathrm{C}$ under $5 \% \mathrm{CO}_{2}$.

\section{Construction of reporter luciferase vectors}

The MafB luciferase reporter constructs -6500/LUC were kindly provided by Dr Masaharu Sakai (Huang et al. 2000). The pCpGfree vector (InvivoGen, San Diego, CA, USA) was digested with SpeI and HindIII at the multi-cloning site (MCS), and the promoters for rat MafB (UR6 region), rat
Ins2, rat Glut2, and rat Srpx2 were cloned using the PCR products generated with the primers listed in Table 1 and the In-Fusion System (Clontech). To clone the human EF1 $\alpha$ core promoter, pCpGrich-mcs (InvivoGen) was digested with SpeI and HindIII. The fragment was cloned into the pCpGfree vector digested with SpeI and HindIII using a Quick Ligation Kit (New England BioLabs, Ipswich, MA, USA). The sequences of the inserts were confirmed by sequencing with the universal RVprimer3 (5'-CTAGCAAAATAGGCTGTCCC-3').

\section{Quantitative RT-PCR analysis of cultured cells}

Quantitative RT-PCR (qRT-PCR) analysis was performed as described previously (Eto et al. 2014). Briefly, total RNA was extracted from the cultured cells using the QIAshredder and RNeasy Mini Kit (Qiagen) according to the manufacturer's instructions. The concentration of purified RNA was measured by a NanoDrop ND1000 Spectrophotometer (Thermo Scientific). RT was performed using High-Capacity cDNA RT Kits (Applied Biosystems). QPCR amplification was performed using the TaqMan Universal PCR Master Mix Core Reagent Kit (Applied Biosystems) with the probes listed in Table 2 and was analyzed using ABI Prism 7900 HT and StepOnePlus (Applied Biosystems); $C_{\mathrm{t}}$ values were measured in duplicates. The mRNA was quantified with normalization to $\beta$-actin expression using the $2-\Delta \Delta C$ t method. The data are presented as the means \pm s.E.M., and statistical significance was determined using a two-tailed unpaired Student's $t$-test. $P<0.05$ was considered to be significant.

\section{In vitro methylation of plasmid DNA}

The pCpG-free vectors were methylated using SssI methylase (New England BioLabs) according to the manufacturer's instructions. Briefly, $10 \mu \mathrm{g}$ of plasmid DNA was

Table 1 Primers used for cloning the indicated promoters

\begin{tabular}{|c|c|c|}
\hline Name & Primer sequences & Product size (bp) \\
\hline MafB UR6 promoter & $\begin{array}{l}\text { F: CCAACATGTAACTAGTTCTCTGCCCCGTCTTTCCTAA } \\
\text { R: GTACAATTCTAAGCTTGCGCCGACAGAGACTCCCTACCC }\end{array}$ & 373 \\
\hline Ins2 promoter & $\begin{array}{l}\text { F: CCAACATGTAACTAGTTTTCTGCAGACCTAGCACCA } \\
\text { R: GTACAATTCTAAGCTTTGTTGGAACAATGACCTGGA }\end{array}$ & 333 \\
\hline Glut2 promoter & $\begin{array}{l}\text { F: CCAACATGTAACTAGTAAACCAATGCCTCCACAGTC } \\
\text { R: GTACAATTCTAAGCTTTTGTAGGAGGAGGAGCAAGC }\end{array}$ & 339 \\
\hline Srpx2 promoter & $\begin{array}{l}\text { F: CCAACATGTAACTAGTTTCCTCAGAACACATCTCTGGA } \\
\text { R: GTACAATTCTAAGCTTAAAAGCCCTTCTACTTTAACATTCC }\end{array}$ & 304 \\
\hline Bisulfited pCpGfree & $\begin{array}{l}\text { F: GGAATTTTTGTAGGGTTTATTTAGGA } \\
\text { R: AAAACAAACAACACCTTAATTTCCA }\end{array}$ & \\
\hline
\end{tabular}


Table 2 TaqMan probes used in this study

\begin{tabular}{l}
\hline Gene symbols \\
\hline Ins1 \\
Ins2 \\
MafA \\
MafB \\
Pdx1 \\
Actb
\end{tabular}

\begin{tabular}{l} 
Gene descriptions \\
\hline Insulin 1 \\
Insulin 2 \\
V-maf avian musculoaponeurotic fibrosarcoma oncogene A \\
V-maf musculoaponeurotic fibrosarcoma oncogene family, \\
protein B (avian) \\
Pancreatic and duodenal homeobox 1 \\
Beta-actin
\end{tabular}

\begin{tabular}{l} 
ID \\
\hline Rn02121433_g1 \\
Rn01774648_g1 \\
Rn00845206_s1 \\
Rn00709456_s1 \\
Rn00755591_m1 \\
4352931E
\end{tabular}

Species

$R$. norvegicus

$R$. norvegicus

$R$. norvegicus

R. norvegicus

R. norvegicus

$R$. norvegicus incubated with or without $\operatorname{Sss}(2.5 \mathrm{U} / \mu \mathrm{g})$ in the presence of $160 \mu \mathrm{M}$ of $S$-adenosylmethionine (New England BioLabs) for $2 \mathrm{~h}$ at $37^{\circ} \mathrm{C}$, and the methylase was then inactivated for $30 \mathrm{~min}$ at $65^{\circ} \mathrm{C}$. After purification of plasmid vectors with a NucleoSpin Plasmid QuickPure Kit (Macherey Nagel, Düren, Germany), the plasmids were used for the reporter assay. In parallel, the degree of methylation was examined via bisulfite sequencing. The vectors were linearized with PacI, which was heatinactivated for $30 \mathrm{~min}$ at $65^{\circ} \mathrm{C}$. The linearized vectors were purified again, followed by the bisulfite conversion with an EpiTect Bisulfite Kit (Qiagen). The bisulfited promoters cloned into the MCS of pCpGfree were PCRamplified with the 'bisulfited CpGfree' primers listed in Table 1. The PCR products were cloned into pCR4-TOPO, sequenced and analyzed as described below.

\section{Reporter assay}

Early-passage (conventional) or late-passage (compromised) INS1 cells and HeLa cells were transfected with $1 \mu \mathrm{g}$ of $6.5 \mathrm{~kb}$ mouse $\mathrm{MafB}$ promoter/luciferase vector (-6500/LUC) (Huang et al. 2000) and pRL-TK vector (Promega) as an internal control using Lipofectamine 2000 (Life Technologies) according to the manufacturer's protocol. The medium was changed $24 \mathrm{~h}$ after transfection. At $48 \mathrm{~h}$ after transfection, the firefly and renilla luciferase activities of the cells were analyzed using the Renilla-Glo and the Firefly Luciferase Assay System (Promega) as described previously (Eto et al. 2014). For the methylated reporter assay, the late-passage INS1 cells were transfected with $1 \mu \mathrm{g}$ of in vitro-methylated pCpGfree-reporter vector and $1 \mu \mathrm{g}$ of pGL4.23 vector (Promega) as an internal control. The medium was changed $24 \mathrm{~h}$ after transfection. At $48 \mathrm{~h}$ after transfection, $50 \mu \mathrm{l}$ of the medium was analyzed for luciferase activity using the Renilla-Glo Luciferase Assay System (Promega), and $10 \mu \mathrm{l}$ of the whole cell extract was analyzed for Luc2 luciferase activity using the Firefly Luciferase Assay System. Each sample in a 96-well plate was analyzed for luciferase activity using a luminescence microplate reader (LB962 CentroPRO; Berthold Technologies, Bad Wildbad, Germany) for $5 \mathrm{~s}$ in each well after automatic shaking of the plates for $10 \mathrm{~s}$. The data are presented as the mean \pm S.E.M., and statistical significance was determined using a two-tailed unpaired Student's $t$-test. $P<0.05$ was considered to be significant.

\section{Prediction of CpG islands and methylation-specific PCR}

For CpG island prediction, the rat MafB promoter $(-3000$ to +36 ) was analyzed using MethPrimer (Li \& Dahiya 2002). The predicted CpG islands were as follows: UR1, from -564 to -19 ( $546 \mathrm{bp}$ ); UR2, from -921 to -576 (346 bp); UR3, from -1118 to -1011 (108 bp); UR4, from -1450 to -1286 (165 bp); UR5, from -1776 to -1670 (107 bp); UR6, from -2058 to -1835 (224 bp); UR7, from -2648 to -2535 (114 bp); and UR8, from -2883 to -2764 (120 bp). Among these regions, PCR primers for GC-rich UR3, UR5, and UR7 were unable to be set.

A total of $1 \mu \mathrm{g}$ of genomic DNA was extracted from INS1 cells using a NucleoSpin Tissue Kit (Macherey Nagel). The bisulfite conversion of DNA and methylation-specific PCR (MSP) were performed using an EpiTect Bisulfite Kit (Qiagen) and an EpiScope MSP Kit (Takara Bio, Shiga, Japan) respectively. The primers used for MSP are listed in Table 3. PCR products were analyzed and quantified by MultiNA (Shimadzu Corporation, Kyoto, Japan).

\section{Bisulfite sequencing}

Bisulfite sequencing was used to analyze the following regions: -237 to +61 for UR1A (299 bp), -321 to -106 for UR1B (216 bp), and -2125 to -1787 (339 bp) for UR6. PCR was performed with the bisulfited DNA using primers listed in Table 4. Nested PCR amplified the regions UR1A and UR1B. The PCR products were cloned into pCR4TOPO (Life Technologies), which was used to transform DH5 $\alpha$ or TOP10 competent cells for bisulfited DNA from INS1 cells, or Mach1 (Life Technologies) for the linearized

Published by Bioscientifica Ltd. 
Table 3 Primers used for methylation-specific PCR

\section{Mice}

Rat MafB UR1A

Methylated

Rat MafB UR1A

Unmethylated

Rat MafB UR1B

Methylated

Rat MafB UR1B

Unmethylated

Rat MafB UR2

Methylated

Rat MafB UR2

Unmethylated

Rat MafB UR4

Methylated

Rat MafB UR4

Unmethylated

Rat MafB UR6

Methylated

Rat MafB UR6

Unmethylated

Rat MafB UR8

Methylated

Rat MafB UR8

Unmethylated

\section{Primer sequences}

F: TTTTAGAGTTAAAGAGTCGGAGAGC

R: CCGAAACAAAATAAAAAAACTAACG

F: GTTTTAGAGTTAAAGAGTTGGAGAGTGA

R: CAAAACAAAATAAAAAAACTAACAAA

F: TCGTGTAGTTATTGGTTAATTCGTC

R: ACTTTCTTACTTACAACCGCTCG

F: TGTGTAGTTATTGGTTAATTTGTTGG

R: ACTTTCTTACTTACAACCACTCACA

F: CGTACGATATATATTTATTCGGTTCG

R: CTCTAAATCGAAATTCCCGC

F: GGTATGTATGATATATATTTATTTGGTTTG

R: CCTCTAAATCAAAATTCCCACA

F: TTAGTTAGTAGGATTTGTACGGGTC

R: AACATTTTATAAAAATTAAACCGAA

F: AGTTAGTAGGATTTGTATGGGTTGG

R: AACATTTTATAAAAATTAAACCAAA

F: GTTTGATTTGGTTGTTAGTGTTGC

R: CCAAAATAAATAAATATCGAATTCG

F: GTTTGATTTGGTTGTTAGTGTTGTG

R: AAAATAAATAAATATCAAATTCACT

F: GGAGGGGTTATTTTAGGATAGAAAC

R: AATTATAAAACCCCCAAAAAATACG

F: GGAGGGGTTATTTTAGGATAGAAAT

R: ATTATAAAACCCCCAAAAAATACACT

\begin{tabular}{c}
\hline Product size (bp) \\
\hline 100 \\
100 \\
139 \\
138 \\
263 \\
268 \\
172 \\
170 \\
206 \\
204 \\
202 \\
201
\end{tabular}

plasmid vector, followed by plating. Single colonies were selected, and the bisulfited DNA cloned into pCR4-TOPO was extracted using a NucleoSpin Plasmid QuickPure Kit (Macherey Nagel), followed by sequencing using the universal sequencing primer RVprimer3 or M13 reverse (5'-CAGGAAACAGCTATGAC-3'). The data were analyzed using quantification tool for methylation analysis (QUMA; Kumaki et al. 2008). The data are presented as the mean \pm s.e.m., and statistical significance was determined using a two-tailed unpaired Student's $t$-test. $P<0.05$ was considered to be significant.

\section{Results}

Upregulation of MafB in the late-passage INS1 cells, a compromised $\beta$-cell model

To analyze the expression of $M a f B$ in the compromised $\beta$-cells without the increased population of other endocrine cells, the INS1 $\beta$-cell line was used, because the change in composition of $\beta$ - and $\alpha$-cells would affect these mRNA expression analyses of the islets isolated from diabetic model mice. Late-passage $\beta$-cell lines chronically exposed to high glucose have been utilized as models for

Table 4 Primers used to amplify and sequence the bisulfite-treated genomic region of the MafB promoter

\begin{tabular}{l} 
CpG island \\
\hline UR1A (nested PCR) \\
First PCR \\
Second PCR \\
UR1B (nested PCR) \\
First PCR \\
Second PCR \\
UR6
\end{tabular}

\section{Primer sequences}

F: GTTAGTTTTTTTTATTTTGTTT

R: AAACAACTCTAACCCCATACT

F: TAGTTTTTTTTATTTTTGTTTT

R: TCTAACCCCATACTCAACTC

F: TTTTAGTGATATTAGGAAGT

R: ACCAAACCTTTATCTAAAAA

F: AAGAAAGTTTTAGAGTTAAAGAG

R: CCAAACCTTTATCTAAAAAC

F: TTTAAGGATTTAAGAGGGGAGAAAA

R: CTACCCTACCAAAAAAACTAAAA
Product size $(b p)$

308

299

304

215

339 
glucotoxic $\beta$-cells (Harmon et al. 2005). The mRNA expression of molecules critical for $\beta$-cell function in INS1 cells with a late passage number (Nishimura et al. 2013) was compared with those with an early passage number. QRT-PCR revealed that the expression of Ins1, Ins2, MafA, and $P d x 1$ was significantly decreased in latepassage INS1 cells (Fig. 1A). Interestingly, MafB mRNA was significantly increased, in these late-passage INS1 cells with p185 and p239 (Fig. 1B), similar to compromised $\beta$-cells in diabetic models, but not in INS1 cells with p42. These results suggest that we could consider late-passage INS1 cells as an in vitro model of compromised $\beta$-cells, and these results prompted us to examine the MafB promoter activity in this population of $\beta$-cells. A reporter assay using the $6.5 \mathrm{~kb} \mathrm{MafB}$ promoter in a luciferase vector showed that $M a f B$ promoter activity was indeed increased in latepassage INS1 cells compared with those in early-passage INS1 cells or HeLa cells (Fig. 1C). The increased transcriptional activity of $M a f B$ is also similar to the $\beta$-cells in diabetic model mice (Nishimura et al. 2015).

\section{Lower methylation levels of the MafB promoter in late-passage INS1 cells}

Demethylation of a particular region of the Arx promoter is involved in the increased expression of $\operatorname{Arx}$, another $\alpha$-cell transcription factor, during the conversion of $\beta$ - to $\alpha$-cells (Dhawan et al. 2011), suggesting that epigenetic factors can be involved in the mechanism of increased expression of transcription factors during the conversion of cell types. Thus, methylation status of the MafB promoter in early- and late-passage INS1 cells was analyzed. Based on the previous reports on CpG islands in the Arx promoter (Dhawan et al. 2011), in silico analysis was performed in the rat MafB promoter from the transcription start site to the upstream $-3000 \mathrm{bp}$, and this analysis detected eight $\mathrm{CpG}$ islands, which were termed UR1-UR8 (Fig. 2A). MSP analyses uncovered various levels of methylation in these regions and showed lower methylation levels of two CpG islands, UR1 ( -564 to -19 ) and UR6 ( -2058 to -1835$)$, in late-passage compared to early-passage INS1 cells (Fig. 2B). Bisulfite sequencing of each region in late-passage INS1 cells demonstrated that seven CpG sites in UR6 were undermethylated compared with early-passage INS1 cells (Fig. 3A, B and C; Supplementa1 Figure 1A, see section on supplementary data given at the end of this article), while no change in UR1 was seen (data not shown). These seven CpG sites were conserved in human, mouse and rat MafB promoters (data not shown). A QUMA revealed that
A mRNA expression in INS1 cells (relative to $\mathrm{p} 24-38$ )

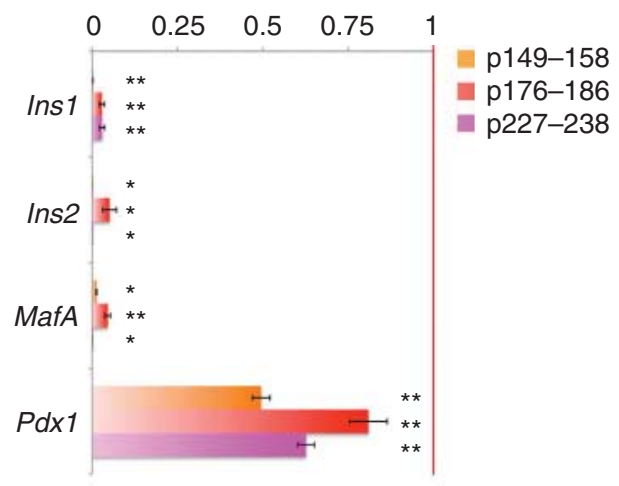

B mRNA expression in INS1 cells (relative to p32)

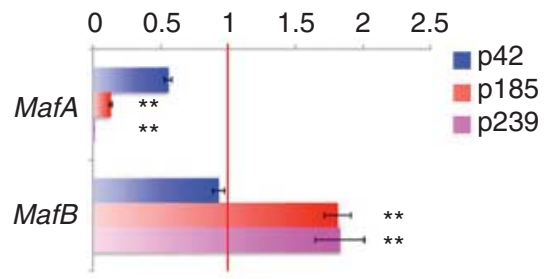

C Relative FLuc activity (with RLuc as internal control)

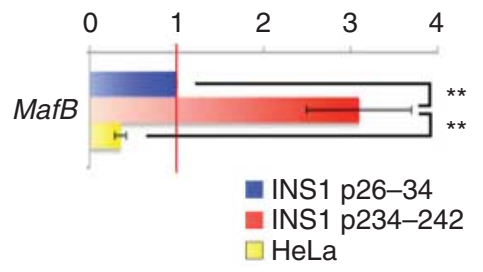

Figure 1

Upregulation of MafB in late-passage INS1 cells. (A) The mRNA expression of the indicated molecules in the INS1 cells with late passage number ( $p 149-158, p 176-186$, and p227-238) relative to early-passage INS1 cells (p24-38) was analyzed by qRT-PCR; $n=3$ for Ins1, Ins2, and MafA; $n=6$ for Pdx1. (B) The mRNA expression of the indicated molecules in the INS1 cells with $p 42, p 185$, and p239 relative to early-passage INS1 cells (p32) was analyzed by qRT-PCR; $n=4$ for MafA and MafB. (C) The reporter assay with the $6.5 \mathrm{~kb}$ MafB/luciferase vector and pRL-TK vector as an internal control, transfected into early-passage INS1 (p26-34; $n=6$ ), late-passage INS1 (p234-242; $n=6)$, and HeLa cells $(n=3)$. The mean \pm s.E.M. are shown $* * P<0.01$ and $* P<0.05$.

$82.0 \pm 7.6 \%, 85.7 \pm 9.3$ and $79.5 \pm 9.1 \%$ of these seven $\mathrm{CpG}$ sites in the UR6 region were methylated in early-passage INS1 cells with p29, p30, and p34, respectively, and $57.2 \pm$ $14.4 \%$ of these CpG sites were methylated in late-passage INS1 cells with p183. Meanwhile, only $39.3 \pm 15.2$ and $39.3 \pm 11.1 \%$ of these CpG sites were methylated in latepassage INS1 cells with p237 and p239-1 (Fig. 3D), which were significantly lower than p29, p30, and p34 INS1 cells respectively (Fig. 3D). Similar results were obtained with

Published by Bioscientifica Ltd. 


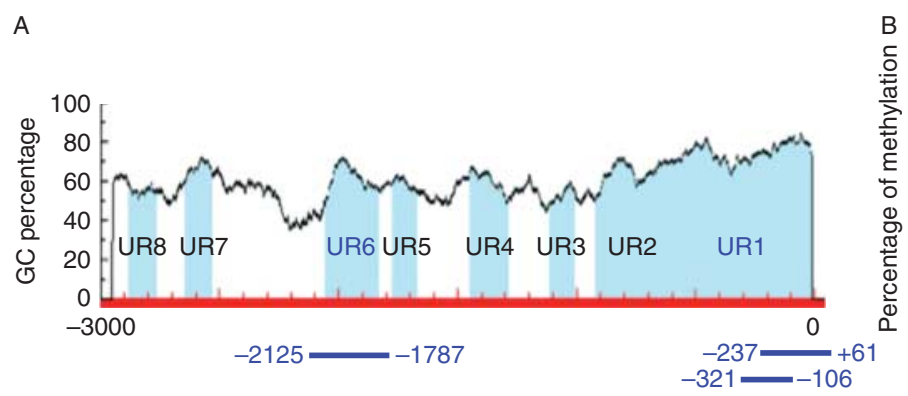

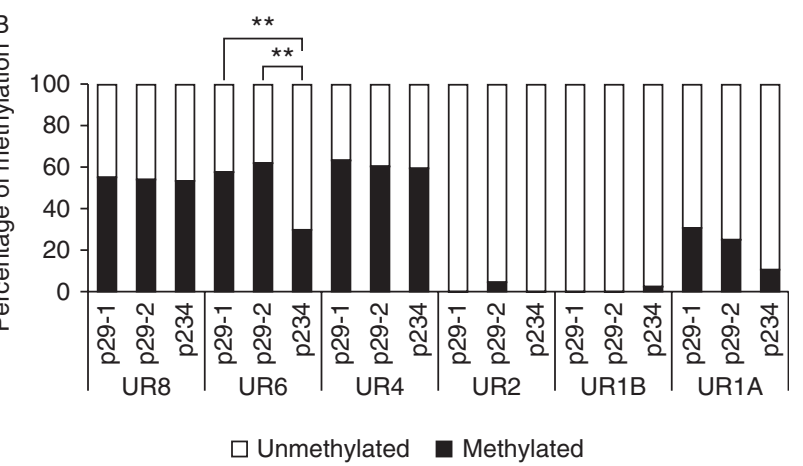

Figure 2

Detection of changes in methylation of the MafB promoter in late-passage INS1 cells. (A) Eight CpG islands in the rat MafB promoter are termed UR1-UR8. Regions underlined in blue were analyzed by bisulfite

other early- and late-passage INS1 cells (Supplementa1 Figure $1 \mathrm{~B}$ and $\mathrm{C}$ ). These results suggest that DNA methylation of the MafB promoter is decreased in the compromised $\beta$-cell model.

\section{Methylation of the UR6 region is involved in the MafB promoter activity in vitro}

Although DNA methylation plays an important role in regulating gene transcription in general, the influence of methylation of individual CpG on the promoter activity of a specific gene remains largely unknown (Hashimoto et al. 2013). The relative contribution of DNA methylation of the UR6 region to the MafB promoter activity was investigated in vitro in late-passage INS1 cells. The effects of methylation on promoter activity can be analyzed by reporter assays after the in vitro methylation of CpGfree luciferase reporter vectors. This pCpGfree vector lacks CpG dinucleotides; therefore, the luciferase activity after the in vitro methylation of this vector is only affected by methylated $\mathrm{CpG}$ residues in the promoter region cloned into the MCS, suggesting its usefulness for studying the effects of $\mathrm{CpG}$ methylation on promoters (Fig. 4A; Klug \& Rehli 2006).

The rat MafB promoter UR6 region (21 CpG in $373 \mathrm{bp}$, GC 57.64\%, Fig. 4B), rat Ins2 promoter (two CpG in 333 bp, GC 53.45\%, Fig. 4C), rat Glut2 promoter (three CpG in 339 bp, GC 50.44\%, Fig. 4D), rat Srpx2 promoter as a negative control (two CpG in 304 bp, GC 45.72\%, Fig. 4E; Øster et al. 2013), and human EF1 $\alpha$ core promoter as a positive control (16 CpG in 221 bp, GC 58.37\%, Fig. 4F; Klug \& Rehli 2006) were cloned into MCS of the pCpGfree reporter vector. In vitro treatment of these vectors with or without DNA methylase SssI was performed, followed by the reporter assay using the late-passage INS1 cells. In sequencing. (B) MSP revealed demethylation of two CpG islands, UR1 $(-564$ to -19$)$ and UR6 ( -2058 to -1835$)$ in the MafB promoter in the late-passage INS1 cells (p234). The mean \pm s.E.M. are shown. $* * P<0.01$.

parallel, linearized vector was analyzed by bisulfite sequencing to examine DNA methylation of the CpG sites in the cloned regions. The luciferase activities driven by unmethylated and methylated Srpx 2 promoter are similar (Fig. 4E), and significantly increased activity of unmethylated EF1alpha promoter was observed (Fig. 4F), both of which were as expected. Results of the methylated reporter assay showed that the promoter activity of unmethylated $M a f B$ promoter UR6 was twice as strong as the promoter activity of methylated UR6 (Fig. 4B), while the methylation of the Ins 2 or Glut 2 promoter, which contain few CpG sites, revealed no changes in promoter activity (Fig. 4C and D). These results revealed that the methylation status of a unique $\mathrm{CpG}$ island in the MafB promoter has an effect on promoter activity in late-passage INS1 cells. The Ins 2 promoter in either methylated or unmethylated form did not exhibit change in the promoter activity, consistent with the previous report that reduced expression of the transcription factors such as MafA or Pdx1 is essential for decreased expression of ins 2 in the compromised $\beta$-cells (Harmon et al. 2005).

\section{Discussion}

In this study, we investigated the mRNA expression of $M a f B$, activity and DNA methylation of the MafB promoter in late-passage INS1 cells, which we considered as a model of compromised $\beta$-cells because the expression of Ins1, Ins 2, MafA, and $P d x 1$ is decreased. In these cells, the mRNA expression and the promoter activity of MafB were increased. Concomitant with these results, the particular region in the MafB promoter UR6 was undermethylated. During development, MafB is expressed in the $\beta$-cells of embryos and early neonates but downregulated in the

Published by Bioscientifica Ltd. 
A

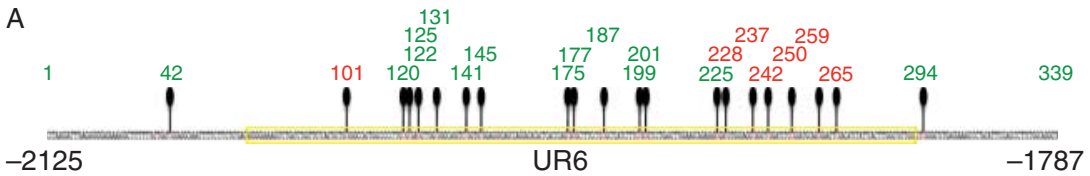

C

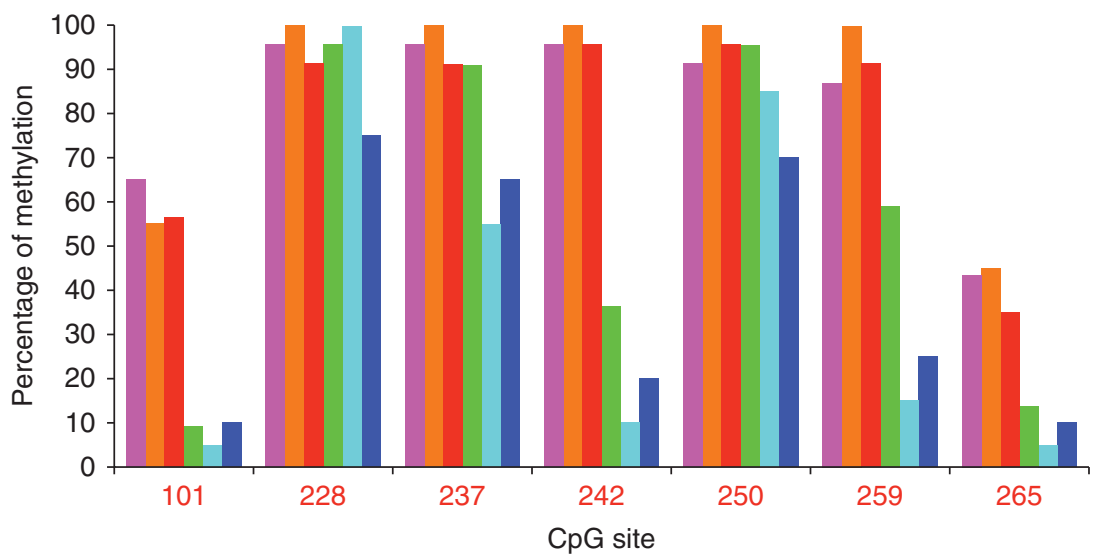

D Averages of \% methylation $(101,228,237,242,250,259,265)$

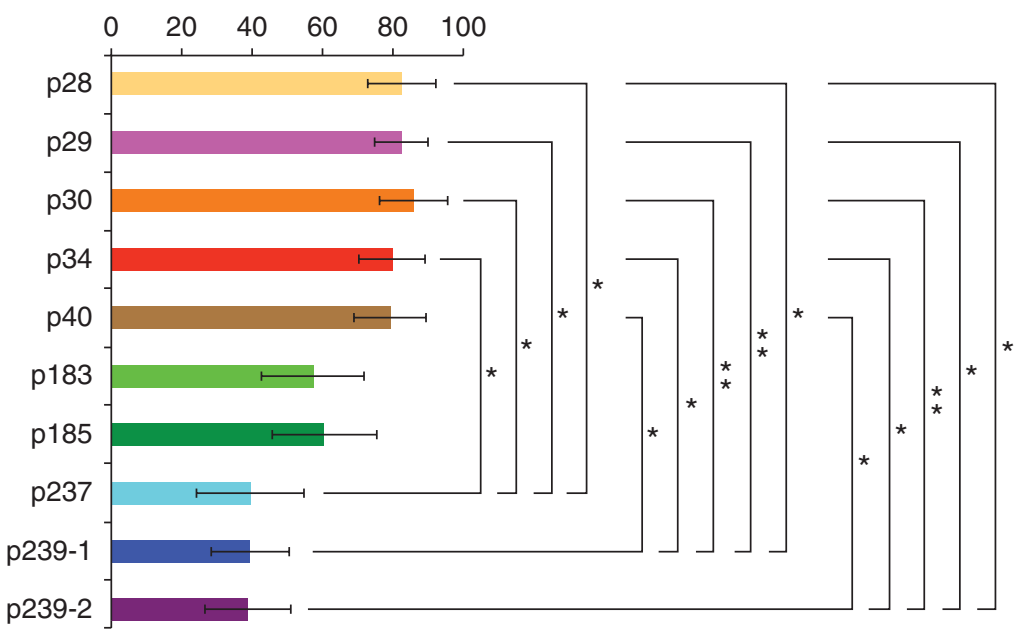

B

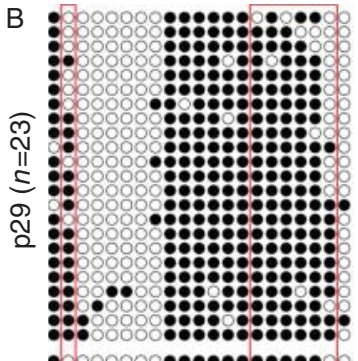

त्र

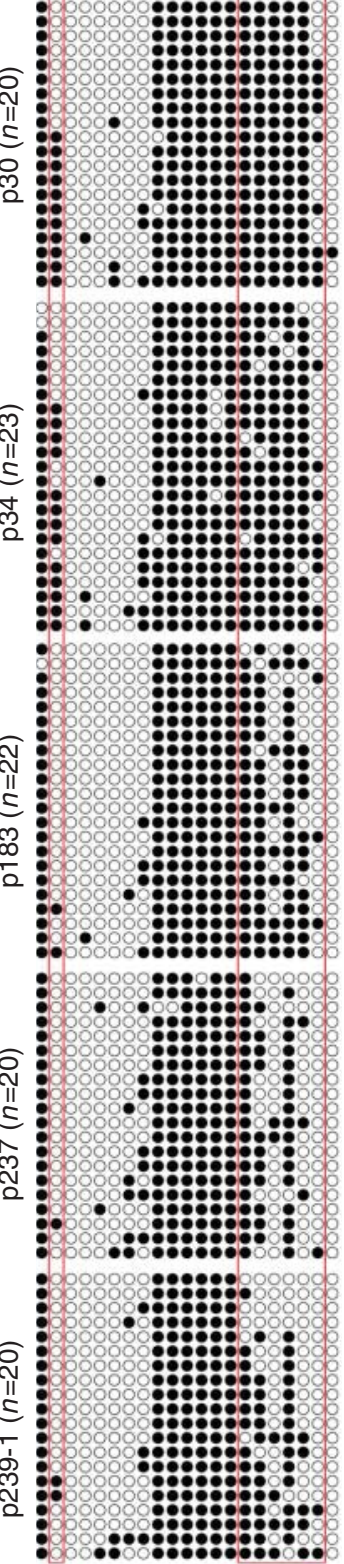

\section{Figure 3}

$\mathrm{CpG}$ islands in the MafB promoter are partially demethylated in latepassage INS1 cells. (A) CPG sites in the UR6 region of the MafB promoter were analyzed by bisulfite sequencing. $\mathrm{CpG}$ islands are highlighted in yellow. The $\mathrm{CpG}$ sites indicated by red numbers were demethylated, and the sites with green numbers were not changed in late-passage INS1 cells compared with early-passage INS1 cells. (B) Analysis of DNA methylation by bisulfite sequencing. $\mathrm{CpG}$ sites demarcated by red squares corresponding

\begin{tabular}{lr}
\hline http://jme.endocrinology-journals.org & (c) 2015 Society for Endocrinology \\
DOI: $10.1530 / \mathrm{ME}-15-0042$ & Printed in Great Britain
\end{tabular}

with the red numbers in (A) were demethylated; $n=23$ for INS1 p29, $n=20$ for INS1 p30, $n=23$ for INS1 p34, $n=22$ for INS1 p183, $n=20$ for INS1 p237, and $n=20$ for INS1 p239-1. (C) Quantification of the results shown in (B). (D) The methylated proportion of $\mathrm{CpG}$ sites corresponding with the red numbers shown in (C) and Supplementa1 Figure 1C. The mean \pm s.E.M. are shown. ${ }^{*} P<0.01$ and $* P<0.05$. 


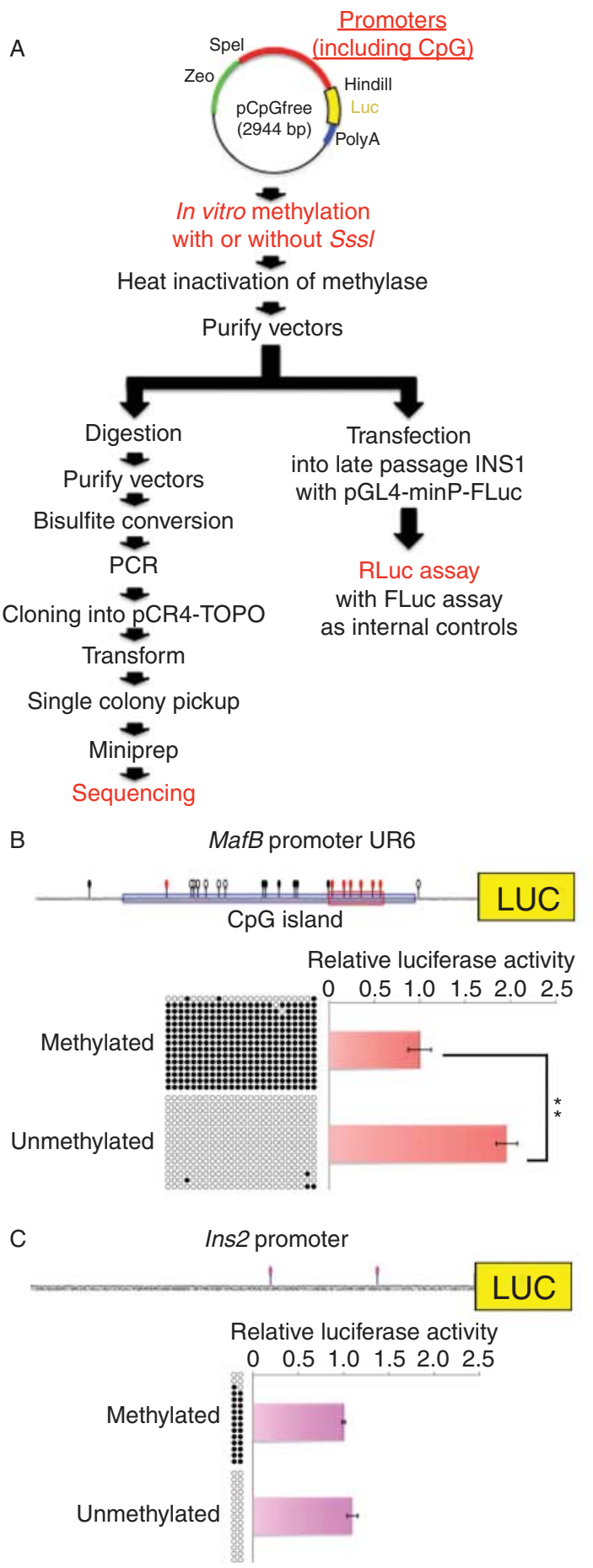

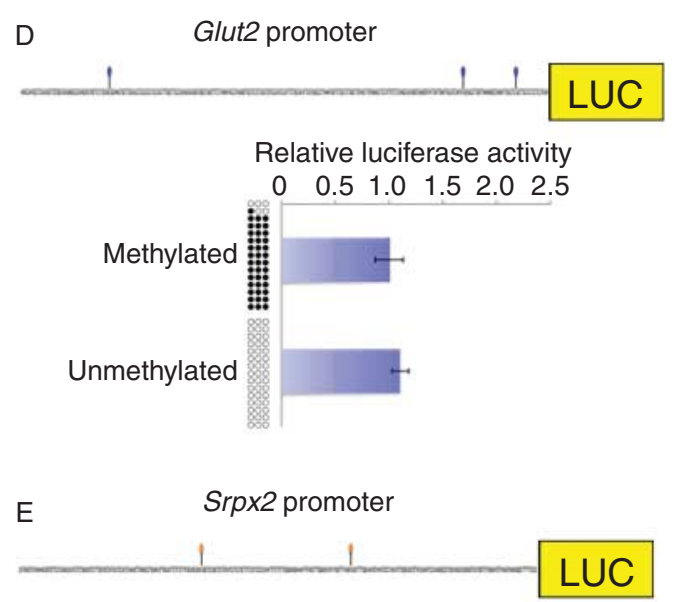

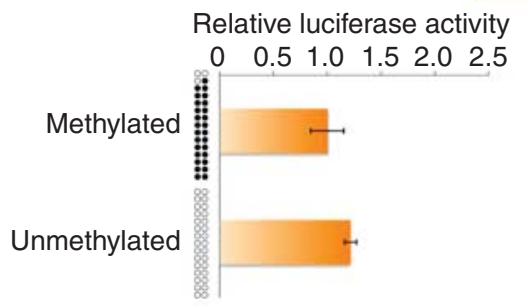

$\mathrm{F}$

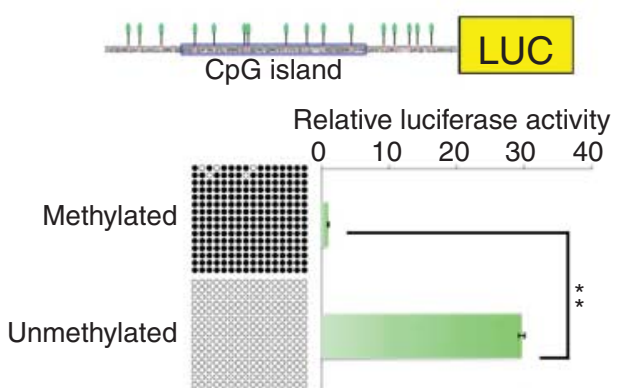

: : CpG site methylated in INS1

9 : CpG site unmethylated in INS1

: CpG site demethylated in late passage INS1

1 9 1 :CpG site

: CpG island

\section{Figure 4}

Methylation of the MafB promoter UR6 is involved in promoter activity in vitro. The experimental protocol $(\mathrm{A})$ explains that the indicated promoters cloned into the $\mathrm{pCpG}$ free vector lacking $\mathrm{CpG}$ dinucleotides were in vitro methylated with or without DNA methylase Sssl, followed by the reporter assay using late-passage INS1 and bisulfite sequencing of those promoter regions, (B) rat MafB (UR6 region), (C) rat Ins2, (D) rat Glut2,
(E) rat Srpx2 (negative control), and (F) human EF1alpha core promoter (positive control). The luciferase activities of unmethylated promoters, treated without Sssl, are shown relative to those of methylated promoters treated with Sssl. The results show the activity of unmethylated MafB promoter UR6 was higher than that of methylated UR6, $n=3$. The mean \pm s.E.M. are shown. ${ }^{*} P<0.01$.

Published by Bioscientifica Ltd 
adult mature $\beta$-cells at least in mice (Nishimura et al. 2006, Artner et al. 2010). Using MafB reporter mice (Moriguchi et al. 2006), we previously reported that the MafB promoter was upregulated in $\beta$-cells of the diabetic model mice (Nishimura et al. 2015). The present study suggests the possibility that MafB upregulation in compromised $\beta$-cells in vivo may also be at least in part due to lower methylation status of the MafB promoter.

The $\beta$-cell line is useful for analyzing DNA methylation, as previously reported in a study describing the demethylation of a particular region of the Arx promoter, which is involved in the upregulation of $\operatorname{Arx}$ during the $\beta$ - to $\alpha$-cell conversion (Dhawan et al. 2011). This is the first study to provide evidence that the partial DNA demethylation of the $M a f B$ promoter can occur in the $\beta$-cell line cultured in a high glucose condition. These results, analyzing the promoters of $\beta$-cell disallowed genes normally repressed in mature $\beta$-cells but upregulated in the compromised $\beta$-cells, suggest that DNA demethylation is a possible mechanism for upregulation of these genes. The larger-scale analysis of $\beta$-cells in the native islets sorted from normal and diabetes model mice by flow-cytometry, which would be physiologically more relevant, may detect other demethylated promoter regions of $\beta$-cell disallowed genes, and MafB may be upstream of these changes in DNA methylation (Vicente-Dueñas et al. 2012) in dedifferentiated $\beta$-cells.

Although changes in the expression level of transcription factors affect gene expression in dedifferentiated $\beta$-cells, our study suggests that the DNA methylation status of gene promoters critical for $\beta$-cell function may play an imperative role. Systematic investigation of epigenetics in $\beta$-cells obtained from normal and diabetes model mice may reveal new mechanisms underlying diabetes development.

In conclusion, increased mRNA expression and promoter activity of $M a f B$ were observed in the compromised $\beta$-cell model of late-passage INS1 cells with reduced expression of Ins1, Ins2, MafA, and Pdx1. The partial demethylation of a specific $\mathrm{CpG}$ island in the MafB promoter was detected in these cells, and the methylation status of this region affected the promoter activity in the reconstituted system. These results suggest that epigenetic regulation may be involved in the molecular mechanism of dysfunction and possible dedifferentiation of pancreatic $\beta$-cells in diabetes mellitus.

\section{Supplementary data}

This is linked to the online version of the paper at http://dx.doi.org/10.1530/ JME-15-0042.
Declaration of interest

The authors declare that there is no conflict of interest that could be perceived as prejudicing the impartiality of the research reported.

\section{Funding}

This study was supported by JSPS KAKENHI (grant numbers 22591022 and 26461351) for W N, grants from the National Center for Global Health and Medicine (22-103, 24-115, and 25-110) for W N and K Y, grants from the Okinaka Memorial Institute for Medical Research, the Takeda Science Foundation, and Japan Diabetes Foundation for W N.

\section{Author contribution statement}

W N contributed to conception and design of the study, the acquisition, analysis and interpretation of the data and drafting the article. N I, K E, N F, $\mathrm{HU}$, and $\mathrm{KY}$ contributed to interpretation of the data and critical revision of the manuscript. $\mathrm{H} \mathrm{M}, \mathrm{S} \mathrm{O}$, and $\mathrm{Y} \mathrm{N}$ contributed to critical revision of the manuscript. All authors have approved the final version, and $\mathrm{W} N$ is responsible for the integrity of the work as a whole.

\section{Acknowledgements}

We thank Drs Claes B Wollheim (University of Geneva, Switzerland) and Nobuo Sekine (Japan Community Healthcare Organization Tokyo Shinjuku Medical Center, Japan) for the INS1 cells and Dr Masaharu Sakai (Hokkaido University, Japan) for the MafB luciferase reporter constructs. We thank Dr Yukiharu Kido, Ms Terue Kikuchi, and Mr Yoshiyasu Nakai for their assistance, and Drs Takao Nammo, Masaki Hiramoto, and Miho Kawaguchi for their helpful discussion.

\section{References}

Artner I, Hang Y, Mazur M, Yamamoto T, Guo M, Lindner J, Magnuson MA \& Stein R 2010 MafA and MafB regulate genes critical to $\beta$-cells in a unique temporal manner. Diabetes 59 2530-2539. (doi:10.2337/ db10-0190)

Asfari M, Janjic D, Meda P, Li G, Halban PA \& Wollheim CB 1992 Establishment of 2-mercaptoethanol-dependent differentiated insulin-secreting cell lines. Endocrinology 130 167-178. (doi:10.1210/endo.130.1.1370150)

Dhawan S, Georgia S, Tschen SI, Fan G \& Bhushan A 2011 Pancreatic $\beta$ cell identity is maintained by DNA methylation-mediated repression of Arx. Developmental Cell 20 419-429. (doi:10.1016/j.devcel.2011.03.012)

Eto K, Nishimura W, Oishi H, Udagawa H, Kawaguchi M, Hiramoto M, Fujiwara T, Takahashi S \& Yasuda K 2014 MafA is required for postnatal proliferation of pancreatic $\beta$-cells. PLoS ONE 9 e104184. (doi:10.1371/ journal.pone.0104184)

Gao T, McKenna B, Li C, Reichert M, Nguyen J, Singh T, Yang C, Pannikar A Doliba N, Zhang T et al. 2014 Pdx1 maintains $\beta$ cell identity and function by repressing an $\alpha$ cell program. Cell Metabolism 19 259-271. (doi:10.1016/j.cmet.2013.12.002)

Harmon JS, Stein R \& Robertson RP 2005 Oxidative stress-mediated, posttranslational loss of MafA protein as a contributing mechanism to loss of insulin gene expression in glucotoxic $\beta$ cells. Journal of Biological Chemistry 280 11107-11113. (doi:10.1074/jbc.M410345200)

Hashimoto K, Otero M, Imagawa K, de Andrés MC, Coico JM, Roach HI, Oreffo RO, Marcu KB \& Goldring MB 2013 Regulated transcription of human matrix metalloproteinase 13 (MMP13) and interleukin-1 $\beta$ (IL1B) genes in chondrocytes depends on methylation of specific proximal promoter CpG sites. Journal of Biological Chemistry $\mathbf{2 8 8}$ 10061-10072. (doi:10.1074/jbc.M112.421156) 
Huang K, Serria MS, Nakabayashi H, Nishi S \& Sakai M 2000 Molecular cloning and functional characterization of the mouse mafB gene. Gene 242 419-426. (doi:10.1016/S0378-1119(99)00500-4)

Klug M \& Rehli M 2006 Functional analysis of promoter CpG methylation using a CpG-free luciferase reporter vector. Epigenetics 1 127-130. (doi:10.4161/epi.1.3.3327)

Kumaki Y, Oda M \& Okano M 2008 QUMA: quantification tool for methylation analysis. Nucleic Acids Research 36 W170-W175. (doi:10.1093/nar/gkn294)

Li LC \& Dahiya R 2002 MethPrimer: designing primers for methylation PCRs. Bioinformatics 18 1427-1431. (doi:10.1093/bioinformatics/18. 11.1427)

Moriguchi T, Hamada M, Morito N, Terunuma T, Hasegawa K, Zhang C, Yokomizo T, Esaki R, Kuroda E, Yoh K et al. 2006 MafB is essential for renal development and F4/80 expression in macrophages. Molecular and Cellular Biology 26 5715-5727. (doi:10.1128/MCB.00001-06)

Nishimura W, Kondo T, Salameh T, El Khattabi I, Dodge R, Bonner-Weir S \& Sharma A 2006 A switch from MafB to MafA expression accompanies differentiation to pancreatic $\beta$-cells. Developmental Biology 293 526-539. (doi:10.1016/j.ydbio.2006.02.028)

Nishimura W, Eto K, Miki A, Goto M, Kawaguchi M, Nammo T, Udagawa H, Hiramoto M, Shimizu Y, Okamura T et al. 2013 Quantitative assessment of Pdx1 promoter activity in vivo using a secreted luciferase reporter system. Endocrinology 154 4388-4395. (doi:10.1210/en.2012-2248) maintenance of the mature $\beta$ cell phenotype in mice. Diabetologia $\mathbf{5 8}$ 566-574. (doi:10.1007/s00125-014-3464-9)
Øster B, Linnet L, Christensen LL, Thorsen K, Ongen H, Dermitzakis ET, Sandoval J, Moran S, Esteller M, Hansen TF et al. 2013 Non-CpG island promoter hypomethylation and miR-149 regulate the expression of SRPX2 in colorectal cancer. International Journal of Cancer 132 2303-2315. (doi:10.1002/ijc.27921)

Pullen TJ \& Rutter GA 2013 When less is more: the forbidden fruits of gene repression in the adult $\beta$-cell. Diabetes, Obesity \& Metabolism 15 503-512. (doi:10.1111/dom.12029)

Scherer WF, Syverton JT \& Gey GO 1953 Studies on the propagation in vitro of poliomyelitis viruses. IV. Viral multiplication in a stable strain of human malignant epithelial cells (strain HeLa) derived from an epidermoid carcinoma of the cervix. Journal of Experimental Medicine $\mathbf{9 7}$ 695-710. (doi:10.1084/jem.97.5.695)

Talchai C, Xuan S, Lin HV, Sussel L \& Accili D 2012 Pancreatic $\beta$ cell dedifferentiation as a mechanism of diabetic $\beta$ cell failure. Cell $\mathbf{1 5 0}$ 1223-1234. (doi:10.1016/j.cell.2012.07.029)

Thorrez L, Laudadio I, Van Deun K, Quintens R, Hendrickx N, Granvik M, Lemaire K, Schraenen A, Van Lommel L, Lehnert S et al. 2011 Tissue-specific disallowance of housekeeping genes: the other face of cell differentiation. Genome Research 21 95-105. (doi:10.1101/gr. 109173.110)

Vicente-Dueñas C, Romero-Camarero I, González-Herrero I, Alonso-Escudero E, Abollo-Jiménez F, Jiang X, Gutierrez NC, Orfao A, Marín N, Villar LM et al. 2012 A novel molecular mechanism involved in multiple myeloma development revealed by targeting MafB to haematopoietic progenitors. EMBO Journal 31 3704-3717. (doi:10.1038/emboj.2012.227)

Received in final form 11 June 2015

Accepted 22 June 2015

Accepted Preprint published online 24 June 2015
(C) 2015 Society for Endocrinology Printed in Great Britain 